\title{
A VISITA DOMICILIÁRIA NA TRANSIÇÃO HOSPITAL-DOMICÍlLIO À PESSOA COM DOENÇA MENTAL - UMA REVISÃO INTEGRATIVA DA LITERATURA
}

\author{
| Soraia Ferreira ${ }^{1}$; Lino Ramos ${ }^{2} \mid$
}

\section{RESUMO}

CONTEXTO: A mudança de um paradigma hospitalocêntrico para um de raiz comunitária vem sendo desde há vários anos contemplada como prioritário. No entanto, continua a existir uma escassa resposta de estruturas necessárias de apoio às pessoas com doença mental e seus cuidadores. O processo de vivência da transição hospital-domicílio pode expor a pessoa a um risco potencial de desenvolver um processo de adaptação desajustado, pelo que a visita domiciliária pode ser um recurso interessante, na medida em que promove a acessibilidade e a aceitabilidade, podendo influenciar positivamente o envolvimento das pessoas e dos seus cuidadores no tratamento destas patologias.

OBJETIVO: Identificar os benefícios da visita domiciliária às pessoas com doença mental na continuidade de cuidados hospital-domicílio. MÉTODO: Realizou-se uma revisão integrativa da literatura, recorrendo-se a pesquisa nas bases de dados EBSCO e B-ON e no RCAAP, tendo resultado em cinco artigos compreendidos no horizonte temporal 2013-2018.

RESULTADOS: A visita domiciliária permite manter o contacto da pessoa com doença mental e os cuidados de saúde, assegura a continuidade de cuidados, demonstrando ter benefícios quer a nível da sintomatologia, da funcionalidade ou da qualidade e vida das pessoas e das suas famílias, reduzindo o desperdício de recursos consumidos durante os reinternamentos por causa das recaídas, além de promover igualmente a sua inserção na comunidade.

CONCLUSÕES: A visita domiciliária deve ser explorada no que concerne aos cuidados a pessoas com doença mental, por ser um recurso que pode diminuir algumas das barreiras identificadas na continuidade de cuidados pós-hospitalar e pelos benefícios que acarreta.

\section{PALAVRAS-CHAVE: Visita domiciliar; Transtornos mentais; Continuidade de assistência ao paciente; Enfermagem psiquiátrica}

\section{RESUMEN}

"La visita a domicilio em la transición hospital-domicilio a la persona com enfermedad mental - una revisión integrativa de la literatura"

CONTEXTO: El cambio de un paradigma centrado en el hospital a uno basado en la comunidad ha sido considerado una prioridad durante varios años. Todavía hay poca respuesta de las estructuras de apoyo necesarias para las personas con enfermedades mentales y sus cuidadores. El proceso de experimentar la transición entre el hospital y el domicilio puede exponer a la persona a un riesgo potencial de desarrollar un proceso de adaptación inapropiado, por lo que la visita a domicilio puede ser un recurso interesante, ya que promueve la accesibilidad, la aceptabilidad y la participación de las personas y sus cuidadores en el tratamiento de estas patologías.

OBJETIVO: Identificar los beneficios de las visitas domiciliarias a personas con enfermedades mentales en la continuidad de la atención hospitalaria domiciliaria.

METODOLOGÍA: Se llevó a cabo una revisión integrativa de la literatura utilizando las bases de datos de EBSCO y B-ON y RCAAP, lo que resultó en la inclusión de cinco artículos en el horizonte temporal 2013-2018.

RESULTADOS: La visita domiciliaria asegura la continuidad de la atención, demuestra beneficios en términos de síntomas, funcionalidad o calidad de vida de las personas y sus familias, reduce el derroche de recursos consumidos, y también promueve su integración en la comunidad.

CONCLUSIONES: Se deben explorar las visitas domiciliarias en relación con la atención de las personas con enfermedades mentales, ya que se trata de un recurso que puede reducir algunas de las barreras identificadas en la continuidad de la atención poshospitalaria y los beneficios que aporta.

\section{DESCRIPTORES: Visita domiciliaria; Trastornos mentales;} Continuidad de la atención al paciente; Enfermería psiquiátrica

\begin{abstract}
"The home visit in the hospital-domicile transition of the person with a mental disorder - an integrative review of the literature"

BACKGROUND: Changing the hospital-centered to a community-based care paradigm has been seen as a priority for a long time. However, there still is a limited response from all the organizations needed to help and support patients with Mental Illnesses and their caretakers. The hospital-home transition and how the patients cope with it might lead to maladjusted adaptation mechanisms. As such, home visits are an important tool that promote accessibility and acceptability, positively influencing patients' and their caretakers' involvment in the treatment of the underlying disease.

AIM: To understand the benefits that home visits can have in promoting the continuity of hospital-home care for the patients with mental illness. METHODS: An integrative literature review was conducted using the EBSCO and B-ON databases and RCAAP, resulting in five articles included in the 2013-2018 time horizon.

RESULTS: The home visit allows the person with mental illness to maintain contact with the health care system, ensures continuity of care, demonstrating benefits in terms of symptoms, functionality or quality of life and life of individuals and their families, reducing the waste of resources consumed during readmissions due to relapses, and also promoting their integration into the community.

CONCLUSIONS: Home visits should be explored with regard to the care of people with mental illness, as this is a resource that can reduce some of the barriers identified in the continuity of post-hospital care and the benefits it brings.
\end{abstract}

\section{KEYWORDS: House calls; Mental disorders; Continuity of patient care; Psychiatric nursing}

Submetido em 30-09-2019

Aceite em 23-12-2019

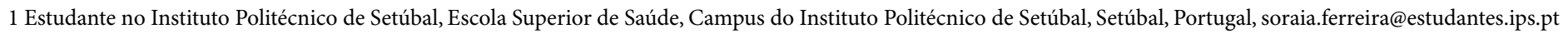
2 Professor Adjunto no Instituto Politécnico de Setúbal, Escola Superior de Saúde, Setúbal, Portugal, lino.ramos@ess.ips.pt

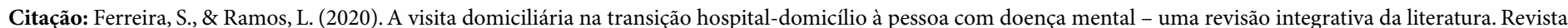
Portuguesa de Enfermagem de Saúde Mental (24), 59-67. 


\section{INTRODUÇÃO}

As perturbações psiquiátricas e os problemas de saúde mental tornaram-se a principal causa de incapacidade e uma das principais causas de morbilidade, nas sociedades atuais (Direção Geral de Saúde [DGS], 2017), pois causam geralmente sofrimento psíquico e perturbam as capacidades relacionais e produtivas de quem delas padece (Almeida, 2018). Segundo este autor, os cuidados de saúde mental na comunidade devidamente organizados com os cuidados de internamento prestados em hospitais gerais podem assegurar um acompanhamento efetivo à quase totalidade dos casos de doença mental grave e apoiar os serviços de cuidado primários a gerir os casos de gravidade ligeira a moderada.

De acordo com a "Pirâmide da organização de serviços para uma combinação ótima de serviços para a saúde mental" (Organização Mundial de Saúde [OMS], 2009), no que concerne a cuidados formais, estes devem ser maioritariamente serviços de cuidados primários para a saúde mental. A desinstitucionalização e o desenvolvimento de cuidados na comunidade são objetivos fundamentais nas políticas da maioria dos países Europeus (DGS, 2017). Os cuidados de saúde primários (CSP) que integrem a saúde mental trazem bastantes benefícios, tendo a OMS (2009) definido razões que o validam, nomeadamente afirmando que os CSP otimizam o acesso das pessoas que necessitam de serviços de saúde mental mais perto das suas casas possibilitando a manutenção das suas atividades quotidianas, assim como a monitorização e gestão a longo-prazo de indivíduos afetados.

No Decreto-Lei no 35/99, de 5 de Fevereiro, alterado e republicado pelo Decreto-Lei n. ${ }^{\circ}$ 304/2009, de 22 de outubro, vem definido sumariamente a organização dos serviços de saúde mental tendo como modelo de referência o comunitário.

Em Portugal, a reorganização dos serviços e reformulação do quadro normativo, tem privilegiado a reabilitação, a reinserção e a integração da saúde mental no sistema de saúde, aparentemente sem grande sucesso (Palha \& Costa, 2015). O Decreto-Lei n. ${ }^{\circ} 8 / 10$ de 28 de janeiro de 2010 veio criar um conjunto de unidades e equipas de cuidados continuados integrados de saúde mental (CCISM), destinado às pessoas com doença mental grave. No entanto, somente decorridos sensivelmente 7 anos desde a publicação deste decreto-lei é que foram definidos os contratos-programa a celebrar com as Unidades e Equipas de Saúde Mental, no âmbito da RNCCI (Despacho n. ${ }^{\circ}$ 1269/17 de 6 de fevereiro de 2017).
Face à escassa resposta de estruturas necessárias de apoio às pessoas com doença mental, os "cuidadores familiares transformaram-se no lastro de sustentação dos programas de saúde mental comunitária" (Almeida, Almeida e Santos, 2010, p.95). É destacado que estes cuidadores podem sofrer de uma elevada sobrecarga objetiva (alteração dinâmica familiar, dificuldades laborais, sociais e financeiras) e subjetiva (sentimentos associados à vivência intrapsíquica desta sobrecarga, com frequentes preocupações com a segurança e futuro dos seus familiares), uma vez que não foram criados mecanismos suficientes que ajudassem a alivar a sua sobrecarga (Xavier e Pereira, 2008 cit in Almeida, Almeida e Santos, 2010). Os mesmos autores (p.111) sugerem o desenvolvimento de "políticas públicas e práticas de atendimento comunitário sensíveis às necessidades dessa população, tais como, a implantação de grupos psico-educativos, visitas domiciliárias regulares e o desenvolvimento de estratégias para enfrentar a sobrecarga que tenha em conta as especificidades de cada família”.

Assim, não somente as pessoas com doença mental, mas também os seus cuidadores, devem ser encarados como parceiros importantes na prestação de cuidados de saúde mental, incentivados a participar nos mesmos e a receber informação e educação, se necessário (Comissão Europeia [CE], 2016).

A acessibilidade é, em todo o mundo, um dos principais problemas no tratamento das doenças mentais e a aceitabilidade também se constitui como outro fator importante na utilização de serviços de saúde mental, uma vez que serviços mais estigmatizantes como os serviços de psiquiatria podem influenciar negativamente a decisão de procurar ou manter cuidados (Almeida, 2018).

Assim, a visita domiciliária (VD) pode ser um recurso interessante, na medida em que promove a acessibilidade e a aceitabilidade, podendo influenciar positivamente o envolvimento das pessoas e dos seus cuidadores no tratamento destas patologias. Assim, procurar-se-á compreender quais os benefícios decorrentes das visitas domiciliárias a pessoas com doença mental e que estratégias devem ser implementadas para o seu sucesso.

\section{MÉTODOS}

Uma revisão integrativa da literatura (RIL) tem como objetivos analisar dados que são extraídos de estudos anteriores e sintetizá-los, fornecendo uma compreensão mais abrangente sobre um determinado fenómeno, constituindo-se assim como um método que proporciona a 
síntese de conhecimento e a incorporação da aplicabilidade de resultados de estudos significativos na prática (Souza, Silva \& Carvalho, 2010), contribuindo para o aperfeiçoamento das práticas baseadas na evidência em Enfermagem. A presente RIL pretende apurar qual a evidência científica existente e de qualidade acerca dos benefícios da VD às pessoas com doença mental e foi regida pelas seguintes etapas: formulação do problema/questão de investigação; extração de resultados; discussão/análise e interpretação dos dados e conclusão. A estratégia PICO, que representa um acrónimo para Patient (participantes/problema), Intervention (intervenção), Control (comparação, contexto), Outcomes (resultados) foi a utilizada para a formulação da questão de investigação que se pretende estudar, tal como evidenciado na seguinte tabela (tabela 1).

Tabela 1 - Critérios utilizados para a formulação da questão de investigação

\begin{tabular}{|c|c|c|c|c|}
\hline $\mathrm{P}$ & $\begin{array}{l}\text { Partici- } \\
\text { pantes }\end{array}$ & $\begin{array}{l}\text { Quem foi } \\
\text { estudado? }\end{array}$ & $\begin{array}{l}\text { Pessoas adultas } \\
\text { com doença } \\
\text { mental }\end{array}$ & \multirow{4}{*}{$\begin{array}{c}\text { DESCRI- } \\
\text { TORES: } \\
\text { Adults with } \\
\text { mental } \\
\text { disorders or } \\
\text { mental illness } \\
\text { Home visits } \\
\text { by nurses } \\
\text { Hospital to } \\
\text { home } \\
\text { Benefits or } \\
\text { advantages } \\
\text { or positive } \\
\text { effects }\end{array}$} \\
\hline I & $\begin{array}{l}\text { Interven- } \\
\text { ção }\end{array}$ & $\begin{array}{l}\text { O que foi } \\
\text { feito? }\end{array}$ & $\begin{array}{l}\text { Visita domicil- } \\
\text { iária }\end{array}$ & \\
\hline $\mathrm{C}$ & Contexto & $\begin{array}{l}\text { Local estu- } \\
\text { dado? }\end{array}$ & $\begin{array}{l}\text { Domicílio após } \\
\text { internamento } \\
\text { no hospital - } \\
\text { continuidade de } \\
\text { cuidados }\end{array}$ & \\
\hline $\mathrm{O}$ & Outcomes & $\begin{array}{l}\text { Resultados, } \\
\text { Efeitos ou } \\
\text { Conse- } \\
\text { quências? }\end{array}$ & $\begin{array}{l}\text { Benefícios da } \\
\text { VD nas pessoas } \\
\text { com doença } \\
\text { mental }\end{array}$ & \\
\hline
\end{tabular}

Foi então estabelecida a seguinte questão de investigação: "Quais os benefícios da visita domiciliária para a continuidade de cuidados hospital-domicílio às pessoas com doença mental?", e na sequência da mesma, definido como objetivo principal: "Identificar os benefícios da visita domiciliária às pessoas com doença mental na continuidade de cuidados hospital-domicílio".

A pesquisa de evidência científica decorreu nas bases de dados EBSCO e B-ON nas revistas CINAHL ${ }^{\oplus}$ Plus, Nursing \& Allied Health Collection, Cochrane Plus Collection, Cochrane Database of Systematic Reviews, Cochrane Methodology Register, MedicLatina e MEDLINE $^{\circledast}$ e no RCAAP (Repositórios Científicos de Acesso Aberto de Portugal), com recurso aos seguintes descritores de pesquisa: "Adults with mental disorders or mental illness", "Home visits by nurses", "Hospital to home" $\mathrm{e}$ "Benefits or advantages or positive effects" com a inclusão do operador boleano/frase "and".
De modo a poder dar resposta à questão de investigação colocada e de modo a orientar a pesquisa realizada foram definidos critérios de inclusão e de exclusão.

Como critérios de inclusão estabeleceram-se os estudos que dessem resposta à questão de investigação referida anteriormente; estudos disponíveis em base de dados com texto na íntegra e revistos por especialistas; estudos compreendidos temporalmente no período decorrido entre janeiro de 2013 e novembro de 2018; estudos nas línguas inglesa, portuguesa ou espanhola; estudos empíricos de natureza qualitativa e quantitativa, assim como revisões sistemáticas e reflexões teóricas. Quanto ao tipo de participantes, os critérios de inclusão estabelecidos são estudos que contemplem pessoas adultas com doença mental no domicílio e/ou profissionais de saúde que realizem visitas domiciliárias a pessoas com doença mental, preferencialmente enfermeiros. Como critérios de exclusão são definidos os estudos que contemplem na sua população crianças e adolescentes (pessoas com idade inferior a 18 anos), pessoas com doença mental que não se encontrem no domicílio.

Quando colocados os critérios de inclusão e exclusão na base de dados EBSCO e no RCAAP não foram encontrados quaisquer resultados, enquanto na base de dados B-On surgiram 28 artigos. Destes, através da leitura do título, foram excluídos 17 artigos, uma vez que a população desses estudos não eram pessoas com doença mental. Assim, restaram 11 artigos, dos quais 2 foram eliminados pela leitura do resumo e 4 foram eliminados após a leitura integral do artigo, pois embora a população desses estudos padecesse de uma doença mental, não se encontravam a residir no seu domicílio. Assim, foram excluídos 23 artigos que não iam ao encontro da questão de investigação colocada.

Totalizaram-se assim 5 artigos que foram incluídos nesta revisão integrativa. Todos apresentam um nível de evidência fidedigno segundo Joanna Briggs Institute (JBI $>50 \%$ ), após terem sido aplicadas as grelhas correspondentes a cada artigo segundo os níveis de evidência JBI - 2 estudos nível de evidência 2c (estudos quasiexperimental); 2 estudos nível de evidência 3 (estudos qualitativos); e 1 estudo nível de evidência 4a (revisão sistemática de estudos descritivos).

A extração dos resultados da amostragem total, através dos quais foi possível realizar a discussão de resultados, encontram-se esquematizados conforme tabela 2. Esta extração de dados foi realizada a partir de grelhas de extração de dados do Joanna Briggs Institute (The Joanna Briggs Institute, 2013). 
Não foi assegurada uma revisão independente, uma vez que apenas os autores deste artigo foram responsáveis pela mesma.

\section{RESULTADOS}

Tabela 2 - Extração de resultados

\begin{tabular}{|c|c|}
\hline $\begin{array}{l}\text { IDENTIFICAÇÃO } \\
\text { DO ESTUDO } \\
\text { (REFERÊNCIA) }\end{array}$ & $\begin{array}{l}\text { OBJETIVO DO ESTUDO; } \\
\text { DESENHO; No E TIPO DE } \\
\text { PARTICIPANTES }\end{array}$ \\
\hline $\begin{array}{l}\text { Chang, Y. \& Chou, } \\
\text { F. (2015). Effects of } \\
\text { Home Visit Interven- } \\
\text { tion on Re-hospi- } \\
\text { talization Rates in } \\
\text { Psychiatric Patients. } \\
\text { Community Ment } \\
\text { Healt. Springer } \\
\text { Science+Business } \\
\text { Media. 51, pp. 598- } \\
605 .\end{array}$ & $\begin{array}{l}\text { - Examinar o impacto da visi- } \\
\text { tação domiciliária na taxa de } \\
\text { reinternamentos e nos custos } \\
\text { médicos em pessoas com es- } \\
\text { quizofrenia e outras perturba- } \\
\text { ções psiquiátricas. } \\
\text { - Estudo retrospetivo. Entre } \\
\text { junho de } 2005 \text { a junho de } 2012 . \\
\text { Participantes diagnosticados } \\
\text { com patologias psiquiátricas } \\
\text { após alta hospitalar, recrutados } \\
\text { do maior hospital no sul de } \\
\text { Taiwan (China), com idades } \\
\text { compreendidas entre os } 20 \text { e os } \\
85 \text { anos. Para o estudo foram } \\
\text { criados } 3 \text { grupos de compara- } \\
\text { ção: } 1 \text { grupo que não recebeu } \\
\text { VD’s (1100 participantes); } 1 \\
\text { grupo com VD’s < } 4 \text { vezes (62 } \\
\text { partic.); } 1 \text { grupo com } 4 \text { ou + } \\
\text { VD’s (179 participantes). }\end{array}$ \\
\hline
\end{tabular}

Gomis, O.; Palma, C. \& Farriols, N. (2017). Intervencion domiciliaria en psicosis: una revision sistemática. Actas Esp Psiquiatria 45(6), pp. 290-302

RESULTADOS
- Em relação ao número de pessoas que foram re-
hospitalizadas existiu uma enorme diferença entre
os grupos: nas pessoas com VD’s <4 ou sem VD’s
62,57\% foram reinternadas; nas pessoas com 4 ou +
VDs a percentagem ficou-se nos $10,61$.
- A taxa de reinternamentos diminuiu assim de
$42,1 \%$ para $11,6 \%$ após VD’s;
- O número de dias de hospitalização e os custos as-
sociados foram menores no grupo com 4 ou + VD’s;
- Género e estado civil não contribuíram para a
taxa de reinternamentos e o número total de dias de
internamento;
- Pessoas mais idosas tiveram menor taxa de rein-
ternamento, mas mais dias de hospitalização;
- Pessoas com mais de 12 anos de escolaridade
tiveram menor taxa de re-hospitalização, dias de
hospitalização mais curtos e menores custos as-
sociados;
- Tipo de intervenção: avaliação de sintomas;
efeitos secundários da medicação; administração da
medicação oral ou de longa duração; obter maior
compreensão da realidade da pessoa e família, saúde
física, estado emocional, dinâmica familiar, situação
económica e social, relações interpessoais ou fatores
de “stress”.
- A intervenção domiciliaria

Analisar os diferentes tipos de serviços domiciliários e propor recomendações para a criação de um serviço que satisfaça as necessidades tanto para doentes com um primeiro episódio psicótico como para doentes psiquiátricos resistentes a tratamento farmacológico e psicológico.

Comparam a intervenção domiciliária na psicose tendo em conta várias tipologias e os seus benefícios.

Fazem-no através de uma revisão sistemática da literatura, considerando 93 artigos (4 revisões sistemáticas e o resto artigos em revistas científicas ou livros de caracter científico) no período compreendido entre 2005-2015.
- A intervenção domiciliária não só tem sido aplicada nos serviços de intervenção precoce da psicose mas também: Intervenção em Crise em Casa - vários estudos, mesmo os mais recentes, demonstraram evitar hospitalização ou reduzir a sua durabilidade. Intervenções incluem a pessoa e a família. Embora a teoria fale em equipas multidisciplinares, a realidade demonstra que costumam ser enfermeiros psiquiátricos a fazerem este serviço. Observadas melhorias a nível da psicopatologia, qualidade de vida, adesão ao tratamento e satisfação relativa aos cuidados prestados; Abordagem através Diálogo Terapêutico (Open Dialogue Approach) - principal objetivo é tratar doentes psicóticos nas suas casas, com uma equipa multidisciplinar. Todo o tipo de intervenção é feita nesse contexto, a partir do diálogo terapêutico. $\mathrm{O}$ tratamento baseia-se na compreensão da doença pelo doente e família, havendo sempre uma avaliação das mudanças que vão ocorrendo. Benefícios a nível da sintomatologia, funcionalidade e redução da terapêutica psicótica. Treino de Habilidades Sociais em âmbito domiciliário a pessoas com esquizofrenia - ao realizar este tipo de intervenções no domicílio, houve uma melhoria das atividades de vida diária, adesão ao tratamento e melhoria da sintomatologia; uso do reforço positivo, modulagem, exercícios ou tarefas em casa e role playing.

- Outras intervenções domiciliárias referidas: contacto telefónico de $2 / 2$ semanas durante $3 \mathrm{~m}$ reduziu atividade alucinatória e recaídas.
CONCLUSÕES

- O principal objetivo das VD's é manter o contacto da pessoa com doença mental e os cuidados de saúde, reduzindo o desperdício de recursos consumidos durante os reinternamentos por causa das recaídas; - Como o número de reinternamentos entre os grupos que não tiveram VD's ou tiveram em número inferior a 4 vezes foram semelhantes sugere que a eficácia de um acompanhamento inadequado de VD's ou não existirem VD's é semelhante (no entanto, os que receberam VD's tiveram custos inferiores);

- $\mathrm{O}$ intervalo médio entre as visitas foi de 32,85 dias;

- Este estudo indica que as VD's fornecem um modelo que assegura a continuidade de cuidados, em que a taxa de reinternamento, o $\mathrm{n}^{\circ}$ de dias de internamento e os custos associados foram menores comparativamente ao grupo que não teve VD'.

- A Abordagem através Diálogo Terapêutico coloca o enfoque na pessoa com doença mental, tentando compreender e dar sentido à experiência sentida, intervindo de forma personalizada. É o tipo de intervenção que tem mais em conta o presente e as potencialidades de toda a rede de suporte (amigos, vizinhos, rede social);

- É fundamental a integração das famílias numa intervenção conjunta; - Para promover a adesão ao tratamento, é importante a vinculação afetiva do doente com a equipa domiciliária;

-Limitações: recusa da família em receber VD's ou a falta de apoio familiar;

- Concluída a intervenção domiciliária pode ser importante o follow-up cara-cara ou telefone, durante pelo menos 6 meses ;

-As intervenções domiciliárias podem melhorar a adesão ao tratamento, a qualidade de vida, habilidades sociais e as relações familiares no meio ambiente natural do doente, reduz hospitalizações e promove a inclusão na comunidade. 
Gleason, H. \& Coyle, E. (2015).

Mental and

behavioral health

conditions among

older adults:

implications for

the home care

workforce. Aging

\& Mental Health.

20 (8), pp. 848-

855
Identificar os desafios que os profissionais enfrentam na prestação de cuidados no domicílio a pessoas com doença mental, bem como as estratégias e apoio que utilizam.

-Entrevistas qualitativas. Foram criados 5 grupos com profissionais (49) que atuavam no domicílio de pessoas com DM (2 grupos tinham formação na área e 3 não) em Massachusetts (EUA), que foram questionados através de entrevistas que foram gravadas em áudio e transcritas textualmente (existiu sempre um moderador nos grupos que usou um protocolo semi estruturado).
- As pessoas de quem cuidavam tinham predominantemente patologias obsessivas (de acumulação), abuso de substâncias e depressão. Outros diagnósticos como esquizofrenia, bipolaridade ou ideação suicida também foram contemplados;

- Independentemente do diagnóstico emergiram tópicos comuns que serviam como barreiras ou facilitadores durante a prestação de cuidados: o comportamento dos doentes, que foram agrupados em 3 categorias: agressiva, disruptiva e psicótica; a comunicação; estratégias de coping e a informação.

- Comunicação: comunicação verbal exprimindo a empatia; importância do silêncio para evitar conflitos, importância do não-verbal, construção da relação interpessoal;

- Estratégias de coping: definir limites na relação; lidar com as próprias emoções; sentimento de realização/dever cumprido; saber como agir em situações de risco que comprometam a segurança do profissional; importância do suporte formal e externo; - Informação: profissionais consideram não receber informação prévia suficiente acerca da pessoa com DM; dinâmicas familiares complicadas que não são facilitadoras; falta de informação/treino na área.
- É destacada a importância de reconhecer os sinais de alerta e "responder" perante o comportamento e não o diagnóstico; - Os resultados do estudo corroboram a ideia de que a consistência do mesmo profissional nas visitas domiciliárias promove a construção da relação terapêutica, a satisfação de ambos e a promoção da qualidade de vida do cliente;

- Em situações de agudização da doença são necessários apoios da polícia ou de outros membros da equipa de saúde;

- Ter as habilidades e as competências para agir prontamente nestas situações e a capacidade de resolução de problemas, torna-se fundamental para o sucesso dos cuidados na comunidade;

-A partilha entre pares emergiu como um recurso importante para validação e partilha de estratégias;

- A importância da formação contínua e desenvolvimento profissional para dar resposta às necessidades. mistos - questionários

Lakeh, M.; Yaghoubi, M; Hajebi. A; Malakouti, SK. \& Vasfi, MG (2017). Costeffectiveness of aftercare services for people with severe mental disorders: an analysis parallel to a randomised controlled clinical trial in Iran. Health and Social Care in the Communitty, 25(3), pp.1151-1159
- Avaliar as preferências das pessoas com doença mental por uma ampla gama de serviços pós-hospitalares e quais as barreiras percecionadas pelos mesmos à continuidade de cuidados pós-internamento.

- Estudo de métodos (242 pessoas) e entrevistas qualitativas (25 pessoas). As preferências foram avaliadas pedindo às pessoas que indicassem os serviços desejados a partir de uma lista de verificação de serviços atualmente disponíveis. A população foi constituída por doentes com alta há 2-4 semanas, de 2 unidades de internamento psiquiátricas (veterans affairs medical centers, que fazem parte do sistema nacional dos EUA com serviços integrados de saúde mental).

- Entre os serviços existentes, o serviço mais referido foi o aconselhamento individual (23,7\%), seguido de acompanhamento domiciliar $(16,2 \%)$ e grupos de apoio $(14,4 \%)$;

- Numa das perguntas abertas feitas na entrevista "O que ajudaria depois da hospitalização?", 18 pessoas responderam “follow up por telefone" e 4 referiram visitas domiciliárias;

- A maioria das pessoas referiu preferir serviços "ao vivo, em pessoa" $(77,4 \%)$, ao invés de por telefone $(13,1 \%)$ ou via vídeo na internet $(1,5 \%)$ - impessoal e receio da questão da privacidade; - Reconsideram o telefone/internet em caso de não haver outra opção ou entre as visitas "pessoalmente";

- Como barreiras que complicavam a continuidade de cuidados pós hospitalar, as pessoas identificaram: problemas de transporte (44,3\%); falar sobre questões que os perturbam e provocam "sintomas desagradáveis" como ansiedade $(36,4 \%)$; motivação $(35,7 \%)$ ou estigma (o facto de familiares ou amigos saberem, $24,7 \%)$;

-O apoio crescente da família/amigos foi considerado como o recurso que mais os ajuda no seu tratamento no pós-alta; seguindo-se do apoio dos pares (outras pessoas doentes) e em terceiro as visitas domiciliárias;

- Várias pessoas referiram que enquanto estavam internadas, lhes falaram em inúmeros serviços, mas que efetivamente nenhum chegou até eles.

- Sintomas que poderão interferir na adesão a estratégias no pós-alta devem ser avaliados e ensinados: autoidentificação de sinais precoces de recaída e os familiares ou amigos estarem atentos a sinais precoces que possam levar a pessoa ao abandono do tratamento.

- Avaliar a relação custointernamento, incluindo acompanhamento telefónico ou visita domiciliária para todas as pessoas durante os 20 meses após a alta hospitalar

-Utilizados para comparação dois grupos de 60 pessoas recrutadas entre 2010 e 2012, que tivessem doença patologia esquizoafetiva ou p.bipolar) e que tivessem estado internadas no Irão. eficácia dos serviços de pósmental grave (esquizofrenia,
- Todas as pessoas do grupo de intervenção receberam: 1. visitas domiciliárias (para os que tinham fraca adesão ao tratamento) ou acompanhamento por telefone, com possibilidade de troca entre as intervenções durante o período de seguimento, 2.educação para a saúde aos cuidadores das pessoas com DMG e 3.treino de habilidades sociais às pessoas.

-O grupo de controlo não foi intervencionado, seguindo apenas as recomendações "normais" que eram feitas no hospital no pósalta;

-A eficácia da intervenção foi medida através de várias escalas que foram aplicadas na admissão do internamento, na alta, $\mathrm{e}$ aos 3,6,12 e 20 meses após a alta e os custos obtidos através de uma equação, em que o número de reinternamentos e a sua duração foram incluídos para os poderem calcular.Nas avaliações periódicas de cuidados pós-alta, todos os indicadores de psicopatologia, qualidade de vida e satisfação com os serviços grupo de controlo. foram melhores no grupo de intervenção comparativamente ao
- Mais de dois terços $(69,1 \%)$ dos entrevistados tiveram pelo menos uma preferência por um tratamento que não estavam a receber atualmente como parte de seu acompanhamento pós-internamento sugere lacunas substanciais nos cuidados; - A maioria preferia ter aconselhamento pessoal;

- Nas entrevistas qualitativas, as pessoas referiram os telefonemas ou visitas domiciliárias como estratégias que preferiam de uma forma "check in", que poderiam ocorrer menos do que uma vez por semana; - A dificuldade no transporte foi a barreira mais mencionada, pelo que contribui para um inadequado tratamento no pós alta;

- Terapia via telefone ou vídeo não parecem ser estratégias que sejam da sua preferência, "aconselhamento pessoal" para mais pessoas na comunidade pode ser inviável em termos e custos, pelo que deve considerar-se as visitas domiciliárias menos frequentes (ou a monitorização telefónica em ambulatório), e que as pessoas preferem.

- Custos de cuidados na comunidade são inferiores quando comparados com os custos hospitalares, pelo que diminuir o número de internamentos diminui o encargo financeiro imposto ao sistema e ao doente e sua família (mais de $2 / 3$ dos custos diretos);

- Reinternamentos diminuíram;

- Maior custo-benefício em intervenções comunitárias como VD's ou acompanhamento por telefone. 


\section{DISCUSSÃO}

Concluída a extração e análise de resultados dos cinco artigos selecionados é possível dar resposta à questão de investigação previamente elaborada: "Quais os benefícios da visita domiciliária na continuidade de cuidados hospital-domicílio às pessoas com doença mental?". De acordo com Chang \& Chou (2015), antes das pessoas internadas terem alta hospitalar, deve ser-lhes facultado, se necessário, um plano que inclua reabilitação, os encaminhamentos e follow ups necessários, devendo esta diretriz ir ao encontro da promoção de intervenções comunitárias nas pessoas com doença mental, como é o caso da intervenção dos profissionais em visitas domiciliárias.

Devido à falta de insight em relação à sua própria patologia, estas pessoas têm um risco acrescido de abandonarem o tratamento contínuo, quer seja psicoterapêutico ou farmacológico, resultando daí múltiplas recaídas que afetam as suas vidas em diferentes dimensões, aumentando a sua dependência e reduzindo a sua funcionalidade (Chang \& Chou, 2015; Gomis, Palma \& Farriols, 2017).

As descompensações psicóticas precoces podem ter um impacto negativo maior, uma vez que podem interferir nas tarefas de vida que são tipicamente completadas durante o fim da adolescência/ início da vida adulta (como o término da escolaridade obrigatória, início da vida profissional ou o estabelecimento de relações sociais extra-família), o que consequentemente resultará em pior prognóstico e maiores custos relacionados com os cuidados de saúde (Chang \& Chou, 2015).

Indo também ao encontro deste facto, Gomis, Palma \& Farriols (2017), elaboraram uma revisão sistemática da literatura acerca da intervenção domiciliária na psicose, analisando os diferentes tipos de serviços domiciliários, de modo a poderem propor recomendações para a criação de um serviço que satisfizesse tanto as necessidades para pessoas com um primeiro episódio psicótico como para doentes psiquiátricos resistentes a tratamento farmacológico e psicológico. Fazem um enfoque especial nas pessoas com um primeiro surto psicótico pois constataram que o período temporal entre o primeiro surto e o tratamento efetivo da doença era demasiado extenso, pelo que intervir precocemente (nos primeiros 3-5 anos, desde a primeira manifestação), melhoraria o prognóstico a longo prazo destes doentes. Estes autores fizeram assim uma comparação entre diferentes intervenções domiciliárias na psicose.
Ressalvam que tão importante como a abordagem à pessoa com doença mental, é a integração das famílias numa intervenção conjunta e independentemente da tipologia, as intervenções domiciliárias demonstraram ter benefícios, nomeadamente: melhorar a adesão ao tratamento, a qualidade de vida, habilidades sociais e as relações familiares no meio ambiente natural onde estão inseridos, reduzir hospitalizações e promover a inclusão na comunidade.

Além destas, num estudo realizado por Lakeh; Yaghoubi; Hajebi; Malakouti \& Vasfi (2017) também se constatou existir um maior custo-benefício. Realizaram um estudo de modo a avaliar a relação custo-eficácia dos serviços de pós-internamento, incluindo acompanhamento telefónico ou visita domiciliária, além da educação e capacitação de habilidades sociais dos cuidadores para todas as pessoas durante os 20 meses após a alta hospitalar, utilizando para comparação dois grupos de 60 pessoas recrutadas entre 2010 e 2012, que tivessem doença mental grave (esquizofrenia, patologia esquizoafetiva ou p.bipolar), em que as pessoas do grupo de intervenção receberam visitas domiciliárias (para os que tinham fraca adesão ao tratamento) ou acompanhamento por telefone, com possibilidade de troca entre as intervenções durante o período de seguimento, educação para a saúde aos cuidadores das pessoas com DMG e treino de habilidades sociais e em que o grupo de controlo não foi intervencionado, seguindo apenas as recomendações "normais" que eram feitas no hospital no pós-alta.

A eficácia da intervenção foi medida através de várias escalas que foram aplicadas na admissão do internamento, na alta, e aos 3,6, 12 e 20 meses após a alta e os custos obtidos através de uma equação, em que o número de reinternamentos e a sua duração foram incluídos para os poderem calcular. Nas avaliações periódicas de cuidados pós-alta, todos os indicadores de psicopatologia (PANSS - escala de sintomas positivos e negativos; young mania rating scale; escala depressão Hamilton; GAF - escala funcionamento global), qualidade de vida (WHOQOL-BREF) e satisfação com os serviços (questionário satisfação do cliente) foram melhores no grupo de intervenção comparativamente ao grupo de controlo. No que concerne aos custos, embora no grupo de intervenção se tenha gastado primeiramente mais (custos associados às VD's, transporte, pagamento dos profissionais), passados os 20 meses, os custos diretos totais do grupo de intervenção foram menores que o do grupo de controlo e à medida que o tempo ia passado, o "cost saving" do grupo de intervenção ia sendo cada vez maior. 
Os reinternamentos diminuíram e provaram existir um maior custo-benefício em intervenções comunitárias como as VD's ou acompanhamento por telefone. Lakeh et al (2017) recomendam a expansão das intervenções domiciliárias a pessoas com DM.

O estudo realizado por Chang \& Chou (2015) também obteve resultados obtidos similares. No sentido de conseguir examinar o impacto da visitação domiciliária na taxa de reinternamentos e nos custos médicos, realizando um estudo durante 7 anos, em que foram incluídos participantes diagnosticados com patologias psiquiátricas após alta hospitalar, recrutados do maior hospital no sul de Taiwan (China), com idades compreendidas entre os 20 e os 85 anos. Para o estudo foram criados 3 grupos de comparação: 1 grupo que não recebeu visitas domiciliárias (1100 participantes); 1 grupo que recebeu menos de 4 visitações domiciliárias (62 partic.) e 1 grupo que recebeu quatro ou mais visitações (179 partic.).

Após a intervenção, verificou-se que existiu uma enorme diferença entre os grupos, em que $62,57 \%$ das pessoas que não receberam nenhuma ou menos de quatro visitações domiciliárias foram reinternadas (o número de reinternamentos entre estes dois grupos foi semelhante, o que sugere que a eficácia de um acompanhamento inadequado de VD's ou de as mesmas não se realizarem é semelhante), contrariamente às pessoas que receberam mais do que quatro visitações domiciliárias em que a percentagem se situou nos 10,61. Assim, conseguiram que a taxa de reinternamentos diminuísse de 42,1\% para $11,6 \%$ após as visitações domiciliárias. O número de dias de hospitalização e os custos associados foram igualmente menores no grupo com 4 ou mais VD's.

Constataram que o género e estado civil não tiveram uma influência significativa na taxa de reinternamentos ou no número total de dias de internamento, contrariamente às pessoas com mais de 12 anos de escolaridade que apresentaram a menor taxa de re-hospitalização, dias de hospitalização mais curtos e menores custos associados. É curioso que as pessoas solteiras e com menos de 12 anos de escolaridade eram as mais representadas nos três grupos, o que pode demonstrar como referido anteriormente que a escolaridade e o estabelecimento de relações interpessoais acabam por ficar comprometidos quando existem descompensações psiquiátricas e o que leva consequentemente a um pior prognóstico.

O tipo de intervenção realizado nas VD's incluiu psicoeducação, orientação nas atividades de vida diárias, promoção da adesão à terapêutica e treino competências sociais básicas, em que avaliação de sintomas, efeitos secundários da medicação; administração da medicação oral ou de longa duração também eram comuns.

Relatam que era tentado obter maior compreensão da realidade da pessoa e família, da sua saúde física, estado emocional, avaliar a dinâmica familiar, situação económica e social, as relações interpessoais ou os fatores de "stress", o que parece ir ao encontro da "Abordagem através do Diálogo Terapêutico", referida por Gomis, Palma \& Farriols (2017) na sua RSL, em que é colocado o enfoque na pessoa com doença mental, tentando compreender e dar sentido à experiência sentida, intervindo de forma personalizada, ocorrendo sempre uma avaliação das mudanças que vão ocorrendo.

Durante a prestação de cuidados no domicílio a pessoas com doença mental, podem existir fatores que o dificultem ou facilitem, e Gleason \& Coyle (2015) procuraram percebê-lo num estudo que realizaram, em que questionaram profissionais que faziam visitas domiciliárias a este grupo populacional. Independentemente do diagnóstico, emergiram tópicos comuns: o comportamento da pessoa cuidada e a comunicação, as estratégias de coping e a informação que os profissionais detinham. Comportamentos agressivos, disruptivos ou psicóticos dificultam a prestação de cuidados (Gleason \& Coyle, 2015), bem como a recusa da família em receber VD’s ou a própria falta de apoio familiar das pessoas com DM que se constituem como limitações da intervenção domiciliária (Gomis, Palma \& Farriols, 2017).

Questões como a importância da comunicação verbal/ não verbal congruente, a utilização do silêncio para evitar conflitos ou a construção da relação terapêutica em que devem ser definidos limites, foram mencionadas frequentemente pelos profissionais que foram entrevistados como fatores facilitadores, bem como estratégias de coping como o saber lidar com as próprias emoções. É destacada a importância da necessidade de se reconhecerem os sinais de alerta e de se "responder" perante o comportamento e não o diagnóstico, a relevância dos profissionais terem as habilidades e as competências para agir prontamente em situações de agudização e a capacidade de resolução de problemas que se torna fundamental para o sucesso dos cuidados na comunidade (Gleason \& Coyle, 2015).

Além dos profissionais estarem atentos a sinais de alerta, também Pfeiffer et al (2016) destacam que os mesmos devem ser ensinados à própria pessoa e aos familiares e pessoas significativas de modo a se prevenirem descompensações e abandono de tratamento por parte da pessoa com DM. 
Pfeiffer et al (2016) realizaram um estudo de modo a avaliarem as preferências das pessoas com DM por uma ampla gama de serviços pós hospitalares e conhecerem quais as barreiras percecionadas pelas mesmas à continuidade de cuidados pós-internamento. Em termos de serviços existentes a maioria preferia ter um "aconselhamento individual", seguindo-se o acompanhamento domiciliar e posteriormente os grupos de apoio. No entanto, consideraram que o apoio da sua família/amigos se constituía como o recurso que mais os ajudaria no pós-alta, seguindo-se do apoio de pares e em terceiro as visitas domiciliárias. Como barreiras que complicavam a continuidade de cuidados pós-hospitalar, as pessoas identificaram problemas de transporte (44,3\%), falar sobre questões que os perturbam e provocam "sintomas desagradáveis" como ansiedade $(36,4 \%)$; motivação $(35,7 \%)$ ou estigma (o facto de familiares ou amigos saberem, $24,7 \%)$. De destacar que $77,4 \%$ das pessoas questionadas e entrevistadas preferiam serviços "ao vivo/em pessoa”, quando comparados com outras opções como telefone ou via vídeo/ internet e que preferiam que as VD's fossem realizadas de forma "check in" e que poderiam ocorrer menos do que uma vez por semana (Pfeiffer et al, 2016).

Sendo a dificuldade no transporte a barreira mais mencionada pelas pessoas e que contribui para um inadequado tratamento no pós-alta, e o facto das pessoas com DM aparentemente aceitarem de forma geral as VD's, Pfeiffer et al (2016) consideram-nas como um recurso que deve considerar-se.

A partilha entre pares emergiu como um recurso importante para validação e partilha de estratégias utilizadas pelos profissionais que realizam VD's e é destacado por Gleason \& Coyle (2015) a formação contínua e desenvolvimento profissional para se ir conseguindo dar resposta às necessidades das pessoas cuidadas. Os resultados deste estudo corroboram a ideia de que a consistência do mesmo profissional nas visitas domiciliárias promove a construção da relação terapêutica, a satisfação de ambos e a promoção da qualidade de vida do cliente, tal como corroborado por Gomis, Palma \& Farriols (2017) que afirmam a vinculação afetiva da pessoa com doença mental à equipa domiciliária como importante no sucesso deste tipo de intervenção domiciliária. Chang \& Chou (2015) e Gomis, Palma \& Farriols (2017) referem que a área da visitação domiciliária a pessoas com doença mental carece de mais investigação.

\section{IMPLICAÇÕES PARA A PRÁTICA CLÍNICA}

Para a prática clínica e uma vez que a organização dos serviços de saúde mental deve ter como modelo de referência o comunitário, a visita domiciliária constituise como uma intervenção que deve ser explorada no que concerne aos cuidados de enfermagem a pessoas com doença mental, não só pelos benefícios supramencionados, mas por ser um recurso que pode diminuir algumas das barreiras identificadas pelos doentes na continuidade de cuidados pós-hospitalar: a dificuldade de transporte, a motivação ou o estigma, uma vez que fornece os cuidados junto do meio de inserção social da pessoa.

Como intervenções a realizar nas visitas domiciliárias, a psicoeducação, promoção adesão à terapêutica, orientação nas atividades de vida diárias e treino de competências sociais básicas foram algumas das realizadas, devendo o enfoque das mesmas, ser colocado não só na pessoa com doença mental, mas também integrando as pessoas significativas, como a família e amigos. Deve procurar-se sempre compreender e dar sentido à experiência vivida da pessoa com doença mental, intervindo de forma personalizada.

Em contexto domiciliário, o estabelecimento de uma relação terapêutica assume-se como fator importante no sucesso da intervenção, pelo que devem ser adotadas estratégias que a promovam, como é o caso de uma comunicação verbal/não verbal congruente, a utilização eficaz do silêncio ou estratégias de coping. De ressalvar a importância do reconhecimento precoce, não só por parte dos profissionais mas também por parte das pessoas com doença mental e das suas famílias, dos sinais de alerta, de forma a se poderem prevenir descompensações e consequências que daí possam advir.

\section{CONCLUSÕES}

Este trabalho procurou compreender quais os benefícios decorrentes das visitas domiciliárias a pessoas com doença mental, como foi referido anteriormente através da questão de partida.

O principal objetivo da visita domiciliária é manter o contacto da pessoa com doença mental e os cuidados de saúde, sendo um modelo que assegura a continuidade de cuidados, demonstrando ter benefícios quer a nível da sintomatologia, da funcionalidade ou da qualidade e vida das pessoas e das suas famílias, reduzindo o 
desperdício de recursos consumidos durante os reinternamentos por causa das recaídas, além de promover igualmente a sua inserção na comunidade (Chang \& Chou, 2015; Gleason \& Coyle, 2015; Gomis, Palma \& Farriols, 2017; Lakeh et al, 2017).

Os autores são unanimes em considerar a visita domiciliária como benéfica na continuidade de cuidados hospital-domicílio à pessoa com doença mental.

\section{REFERÊNCIAS BIBLIOGRÁFICAS}

Almeida, J.; Almeida, H. e Santos, E. (2010). Sobrecarga dos cuidadores familiares de pessoas com doença mental: dimensões analítico-reflexivas na perspectiva do Serviço Social. Psychologica, 2(52), 91-116. Doi: 10.14195/1647-8606_52-2_5

Almeida, J. (2018). A saúde mental dos portugueses. Lisboa: Fundação Francisco Manuel dos Santos.

Chang, Y. \& Chou, F. (2015). Effects of Home Visit Intervention on Re-hospitalization Rates in Psychiatric Patients. Community Mental Health Journal - Springer Science+Business Media, 51(5), 598-605. Doi: 10.1007/ s10597-014-9807-7

Comissão Europeia [CE] (2016). European Framework for Action on Mental Health and Wellbeing. Bruxelas: CE.

Decreto-Lei n. ${ }^{\circ}$ 8/10 de 28 de Janeiro. (2010). Diário da República nº 191/2010 - Ia Série. Lisboa.

Decreto-Lei no 304/09 de 22 Outubro. (2009). Diário da Republica n² 205/2009 - Ia Série. Lisboa.

Despacho n. ${ }^{\circ}$ 1269/17 de 6 de fevereiro. (2017). Diário da República n 26/2017 - II Série. Lisboa.

Direção Geral de Saúde. (2017). Programa Nacional para a Saúde Mental. Lisboa: DGS.

Gleason, H. \& Coyle, E. (2015). Mental and behavioral health conditions among older adults: implications for the home care workforce. Aging \& Mental Health, 20 (8), 848-855. Doi: 10.1080/13607863.2015.1040725
Gomis, O.; Palma, C. \& Farriols, N. (2017). Intervencion domiciliaria en psicosis: una revision sistemática. Actas Espanholas de Psiquiatria, 45(6), 290-302. Disponível em: https://www.actaspsiquiatria.es/repositorio/19/110/ESP/19-110-ESP-290-302-827522.pdf

Lakeh, M.; Yaghoubi, M; Hajebi. A; Malakouti, SK. \& Vasfi, MG (2017). Cost-effectiveness of aftercare services for people with severe mental disorders: an analysis parallel to a randomised controlled clinical trial in Iran. Health and Social Care in the Communitty, 25(3), 11511159. Doi: $10.1111 /$ hsc. 12416

Organização Mundial de Saúde. (2009). Integração da saúde mental nos cuidados de saúde primários - Uma perspetiva global. Genebra: OMS.

Palha, F. e Costa, N. (2015). Trajetórias pelos cuidados de saúde mental. Parte I - O processo de "desinstitucionalização" psiquiátrica em Portugal: da análise objetiva dos factos às perceções de utentes, familiares/ cuidadores e técnicos. Porto: ENCONTRAR+SE

Pfeiffer, P; Bowersox, N; Birgenheir, D; Burgess, J.; Forman, J. \& Valensteun, M. (2016). Preferences and Barriers to Care Following Psychiatric Hospitalization at Two Veterans Affairs Medical Centers: A Mixed Methods Study. The Journal of Behavioral Health Servces \& Research, 43(1), 88-103. Doi: 10.1007/s11414-015-9460-0

Souza, M; Silva, M. \& Carvalho, R. (2010). Revisão integrativa: o que é e como fazer. Einstein, 8(1), 102-106. Disponível em: https://www.scielo.br/pdf/eins/v8n1/ pt_1679-4508-eins-8-1-0102.pdf

The Joanna Briggs Institute. (2013). The New JBI Levels of Evidence and Grades. Levels of Evidence - Effectiveness. Disponível em: https://joannabriggs.org/jbi-approach.html\#tabbed-nav=Levels-of-Evidence 